\title{
Novel cardiovascular risk biomarkers in metabolic syndrome
}

\author{
Fabrizio Montecucco*,1,2 (iD), Luca Liberale ${ }^{3,4}$ \& Federico Carbone Li, $^{1,4}$ \\ ${ }^{1}$ Ospedale Policlinico San Martino Genoa - Italian Cardiovascular Network, 10 Largo Benzi, Genoa 16132, Italy \\ ${ }^{2}$ First Clinic of Internal Medicine, Department of Internal Medicine \& Centre of Excellence for Biomedical Research (CEBR), \\ University of Genoa, 6 viale Benedetto XV, Genoa 16132, Italy \\ ${ }^{3}$ Center for Molecular Cardiology, Schlieren Campus, University of Zurich, Wagistrasse 12, 8952 Schlieren, Switzerland \\ ${ }^{4}$ First Clinic of Internal Medicine, Department of Internal Medicine, University of Genoa, 6 viale Benedetto XV, Genoa 16132, Italy \\ *Author for correspondence: Tel.: +3901 0335 1054; Fax: +3901 0353 8686; fabrizio.montecucco@unige.it
'Guture research should focus on two key points: further validating diagnostic/predictive values of available and new inflammatory biomarkers, eventually combining them in panel; better understanding the complex regulatory system underlying inflammatory response in Mets"

First draft submitted: 20 August 2019; Accepted for publication: 9 September 2019; Published online: 10 October 2019

\begin{abstract}
Keywords: biomarker $\bullet$ C-reactive protein $\bullet$ epigenetics $\bullet$ inflammation $\bullet$ macrophages $\bullet$ metabolic syndrome $\bullet$ microRNA • neutrophils • obesity • osteopontin
\end{abstract}

Epidemiological data clearly indicate a strong link between obesity, metabolic disorders and cardiovascular (CV) risk factors. Their clustering (usually referred to as metabolic syndrome [MetS]) synergistically acts in conferring an overall CV risk higher than that of each single condition [1]. Despite this evidence, the pathophysiology of MetS still represents a challenge for both scientists and clinicians, as even the role of adipose tissue (AT) is currently questioning. Alongside with the unequivocal link between obesity (as measured by body mass index [BMI]) and increased mortality in the general population, clinical studies in patients with established CVD have paradoxically reported a better outcome in obese individuals [2]. Indeed, the use of BMI per se does not truly consider body fat composition/distribution and then it cannot support pathophysiological relevance of epidemiological observations. Therefore, the paradoxical association between high BMI and better CV outcome, usually referred to as 'obesity paradox' should be actually considered as a 'BMI paradox' [2]. When indexes of abdominal adiposity are used, this paradox disappears: waist circumference and waist-to-hip ratio are indeed linearly associated with increased CV mortality risk [3]. Although genetic variables have a causal role in developing central obesity and related metabolic complications, there is still no explanation for their contribution to molecular and clinical features of MetS, as for their variation across ethnicity and gender [4]. Volumetric studies and secretome analysis are increasingly clarifying how body fat depots contribute to MetS and CV risk through a multitude of dysregulated pathways involving adipogenesis, adipocytokine balance, oxidative stress, lipolysis and inflammation [5-8]. Concerning the latter, the most of the studies focused on infiltrating macrophages and their related mediators. Especially CRP has been widely reported as the classical paradigm of inflammatory biomarker associated with atherosclerotic CV disease.

Several studies also linked CRP with abdominal adiposity and other clinical features of MetS [9]. In line with this, many researches attempted at including serum hs-CRP as additional diagnostic criterion for MetS diagnosis [10]. Nevertheless, its molecular implication in MetS has not yet been elucidated so that neither hs-CRP nor other inflammatory biomarkers are considered as diagnostic criteria by any guideline. Among them, the expression of osteopontin (OPN) was shown to dramatically increase in the AT of obese patients, where promoted adipocyte dysfunction at several levels: impaired pre-adipocyte maturation [11], aromatase expression with estradiol production [12] and even a osseous differentiation of fat tissue [13]. Far from having only a local effect, visceral fat-derived OPN displays a role in promoting hepatic steatosis and cardiac aging through mechanisms involving matrix remodeling, fibrosis and oxidative stress [14-16]. Further supporting an active role in obesity and MetS, OPN was also shown to correlate with clinical response to diet and bariatric surgery $[17,18]$. Nevertheless, at least two critical

Future Medicine 
points about the biological activity of OPN in MetS still remain to be clarified. First, peripheral expression of OPN still remains poorly investigated. Growing evidence indicates that pro-inflammatory polarized immune cells accumulate in skeletal muscle where they alter myocyte insulin sensitivity via paracrine or autocrine effects. Even a direct relationship was not yet confirmed, nonspecific OPN downstream pathways are involved in this process. Especially the activation of IKK/NF- $\mathrm{KB}$ pathway has been described to potentially inhibit downstream insulin signaling at different levels, including the suppression of GLUT4 expression [19]. Recently, immunohistochemistry analyses demonstrated that OPN expression was associated to macrophage infiltration in an experimental model of skeletal muscle injury [20]. Future studies are expected to clarify whether OPN may also have a role in the skeletal muscle metabolism. To date one only preliminary study has associated serum levels of OPN to the improvement of peripheral insulin sensitivity in a small cohort of obese diabetic patients undergoing bariatric surgery [18]. In addition to predict diabetes remission independently duration of disease and weight loss (adjusted OR: 1.05; 95\% CI: 1.01-1.10), serum OPN was also independently associated with the HOMA2-\%S, a surrogate measure of peripheral insulin sensitivity $(\beta=0.738 ; \mathrm{p}<0.001)$. As a second point, the biological activity of OPN in MetS have been so far limited to macrophage proliferation and polarization [21,22], thus forgetting the strong chemotactic activity toward neutrophils. Therefore, while the fingerprints of MetS on neutrophil metabolomic profile have been demonstrated [23], neutrophils are still considered mere effector cells with no direct involvement role in MetS pathophysiology. Only recently macrophage-released OPN has been associated with neutrophil chemotaxis nonalcoholic steatohepatitis [24]. Indeed, transplantation of macrophage-depleted visceral AT into lean recipient mice promoted hepatic macrophage accumulation [24]. As also observed in vitro, this would be due to the enhanced expression of transmembrane adhesion molecules (i.e., CD11c) in AT macrophages [25]. However, only in the context of atherosclerotic plaques, a link between OPN, neutrophil recruitment and local injury (i.e., plaque rupture) was demonstrated [26]. Serum OPN was indeed demonstrated to strongly correlate not only with intraplaque pro-inflammatory M1 macrophage content $(\mathrm{r}=0.187 ; \mathrm{p}=0.009)$, but especially with the extent on neutrophil infiltration $(r=0.421 ; p<0.001)$. Far from being a mere observational finding, serum OPN was also demonstrated to predict plaque rupture and consequent atherothrombotic events independently of age and gender (adjusted HR: 13.1; 95\% CI: 2.84-60.78) [26]. Due to the pleiotropic activity, OPN may be then considered a paradigm of the complex inflammatory processes underlying MetS.

Although it has already been suggested as a CV risk biomarker [27], any potential use in clinical practice shall be preceded by a further understanding of its biological role. Therefore, despite promising like OPN, the clinical utility of biomarkers in MetS remains to be validated and cannot be recommended in the daily clinical practice. Nevertheless, ongoing studies are more in deep exploring inflammatory processes underlying MetS. On the one hand, there is great attention to identify novel biomarkers of MetS. Among them microRNA (miRNA) have aroused global interest due to their ubiquitous distribution in metabolic active tissues such as liver, skeletal muscle, AT and $\beta$ cells [28]. Many of them are involved in regulating the relevant genes and pathways of main components of MetS, therefore showing a diagnostic and therapeutic potential. On the other hand, the molecular signals underlying MetS are constantly under investigation. Especially the epigenetic signature of AT is another intriguing field of research, being widely influenced by nutrition and microbiota [29], Even though a direct association of blood leukocyte DNA methylation with adiposity has not yet been demonstrated, hyperglycemia was shown to upregulate OPN expression by glucose-induced histone acetylation and methylation processes [30]. Finally, it should take into account that future studies have to carefully consider the analysis of gender differences, which plays a leading role in metabolically induced inflammation [31]. Although AT distribution across gender influences the inflammatory response, leukocytes in males are constitutively more prone to be recruited and activated. In line, we have previously reported how gender difference influences both clinical and inflammatory response to bariatric surgery [32]. In conclusion, future research should focus on two key points: further validating diagnostic/predictive values of available and new inflammatory biomarkers, eventually combining them in panel; better understanding the complex regulatory system underlying inflammatory response in MetS.

Financial \& competing interests disclosure

The authors have no relevant affiliations or financial involvement with any organization or entity with a financial interest in or financial conflict with the subject matter or materials discussed in the manuscript. This includes employment, consultancies, honoraria, stock ownership or options, expert testimony, grants or patents received or pending, or royalties.

No writing assistance was utilized in the production of this manuscript. 


\section{References}

1. Papakonstantinou E, Lambadiari V, Dimitriadis G, Zampelas A. Metabolic syndrome and cardiometabolic risk factors. Curr. Vasc. Pharmacol. 11(6), 858-879 (2013).

2. Antonopoulos AS, Tousoulis D. The molecular mechanisms of obesity paradox. Cardiovasc. Res. 113(9), 1074-1086 (2017).

3. Vecchie A, Dallegri F, Carbone F et al. Obesity phenotypes and their paradoxical association with cardiovascular diseases. Eur. J. Int. Med. 48, 6-17 (2018).

4. Stryjecki C, Alyass A, Meyre D. Ethnic and population differences in the genetic predisposition to human obesity. Obes. Rev. 19(1), 62-80 (2018).

5. Carbone F, Mach F, Montecucco F. The role of adipocytokines in atherogenesis and atheroprogression. Curr. Drug Targets 16(4), 295-320 (2015).

6. Liberale L, Bonaventura A, Vecchie A et al. The role of adipocytokines in coronary atherosclerosis. Curr. Atherosclerosis Rep. $19(2), 10$ (2017).

7. Ansaldo AM, Montecucco F, Sahebkar A, Dallegri F, Carbone F. Epicardial adipose tissue and cardiovascular diseases. Int. J. Cardiol. 278, 254-260 (2019).

8. Ferrara D, Montecucco F, Dallegri F, Carbone F. Impact of different ectopic fat depots on cardiovascular and metabolic diseases. J. Cell. Physiol. 234(12), 21630-21641 (2019).

9. Lapice E, Maione S, Patti L et al. Abdominal adiposity is associated with elevated C-reactive protein independent of BMI in healthy nonobese people. Diabetes Care 32(9), 1734-1736 (2009).

10. Devaraj S, Singh U, Jialal I. Human C-reactive protein and the metabolic syndrome. Curr. Opin. Lipidol. 20(3), 182-189 (2009).

11. Moreno-Viedma V, Tardelli M, Zeyda M, Sibilia M, Burks JD, Stulnig TM. Osteopontin-deficient progenitor cells display enhanced differentiation to adipocytes. Obes. Res. Clin. Pract. 12(3), 277-285 (2018).

12. Leitner L, Jurets A, Itariu BK et al. Osteopontin promotes aromatase expression and estradiol production in human adipocytes. Breast Cancer Res. Treat. 154(1), 63-69 (2015).

13. Bondarava M, Cattaneo C, Ren B et al. Osseous differentiation of human fat tissue grafts: from tissue engineering to tissue differentiation. Sci. Rep. 7, 39712 (2017).

14. Lancha A, Rodriguez A, Catalan V et al. Osteopontin deletion prevents the development of obesity and hepatic steatosis via impaired adipose tissue matrix remodeling and reduced inflammation and fibrosis in adipose tissue and liver in mice. PLoS ONE 9(5), e98398 (2014).

15. Sawaki D, Czibik G, Pini M et al. Visceral adipose tissue drives cardiac aging through modulation of fibroblast senescence by osteopontin production. Circulation 138(8), 809-822 (2018).

16. Glass O, Henao R, Patel K et al. Serum interleukin-8, osteopontin, and monocyte chemoattractant protein 1 are associated with hepatic fibrosis in patients with nonalcoholic fatty liver disease. Hepatol. Commun. 2(11), 1344-1355 (2018).

17. Wang $\mathrm{X}$, Cheng $\mathrm{M}$, Zhao $\mathrm{M}$ et al. Differential effects of high-fat-diet rich in lard oil or soybean oil on osteopontin expression and inflammation of adipose tissue in diet-induced obese rats. Eur. J. Nutr. 52(3), 1181-1189 (2013).

18. Carbone F, Adami G, Liberale L et al. Serum levels of osteopontin predict diabetes remission after bariatric surgery. Diabetes Metab. 45(4), 356-362 (2018).

19. Wu H, Ballantyne CM. Skeletal muscle inflammation and insulin resistance in obesity. J. Clin. Invest. 127(1), 43-54 (2017).

20. Wijesinghe DKW, MacKie EJ, Pagel CN. Normal inflammation and regeneration of muscle following injury require osteopontin from both muscle and non-muscle cells. Skelet. Muscle 9(1), 6 (2019).

21. Schuch K, Wanko B, Ambroz K et al. Osteopontin affects macrophage polarization promoting endocytic but not inflammatory properties. Obesity 24(7), 1489-1498 (2016).

22. Tardelli M, Zeyda $\mathrm{K}$, Moreno-Viedma $\mathrm{V}$ et al. Osteopontin is a key player for local adipose tissue macrophage proliferation in obesity. Mol. Metab. 5(11), 1131-1137 (2016).

23. Palomino-Schatzlein M, Simo R, Hernandez C et al. Metabolic fingerprint of insulin resistance in human polymorphonuclear leucocytes. PLoS ONE 13(7), e0199351 (2018).

24. Bijnen M, Josefs T, Cuijpers I et al. Adipose tissue macrophages induce hepatic neutrophil recruitment and macrophage accumulation in mice. Gut 67(7), 1317-1327 (2018).

25. Thomas D, Apovian C. Macrophage functions in lean and obese adipose tissue. Metabolism 72, 120-143 (2017).

26. Carbone F, Rigamonti F, Burger F et al. Serum levels of osteopontin predict major adverse cardiovascular events in patients with severe carotid artery stenosis. Int. J. Cardiol. 255, 195-199 (2018).

27. Van Der Leeuw J, Beulens JW, Van Dieren S et al. Novel biomarkers to improve the prediction of cardiovascular event risk in Type 2 diabetes mellitus. J. Am. Heart Assoc. 5(6), (2016).

28. Huang Y, Yan Y, Xv W et al. A new insight into the roles of MiRNAs in metabolic syndrome. Biomed. Res. Int. 2018, 7372636 (2018). 
29. Stols-Goncalves D, Tristao LS, Henneman P, Nieuwdorp M. Epigenetic markers and microbiota/metabolite-induced epigenetic modifications in the pathogenesis of obesity, metabolic syndrome, Type 2 diabetes, and non-alcoholic fatty liver disease. Curr. Diabetes Rep. 19(6), 31 (2019).

30. Cai M, Bompada P, Atac D, Laakso M, Groop L, De Marinis Y. Epigenetic regulation of glucose-stimulated osteopontin (OPN) expression in diabetic kidney. Biochem. Biophys. Res. Commun. 469(1), 108-113 (2016).

31. Varghese M, Griffin C, Singer K. The role of sex and sex hormones in regulating obesity-induced inflammation. Adv. Exp. Med. Biol. 1043, 65-86 (2017).

32. Carbone F, Nulli Migliola E, Bonaventura A et al. High serum levels of C-reactive protein (CRP) predict beneficial decrease of visceral fat in obese females after sleeve gastrectomy. Nutr. Metabol. Cardiovasc. Dis. 28(5), 494-500 (2018). 